\title{
Helicobacter Pylori Infection Increases the Incidence of Erosive Oral Lichen Planus and Alters the Oral Microbiome Composition
}

\section{Yangheng Zhang}

Nanjing University

Shutong Li

Shandong University

Jingyuan Li

Shandong University

Ya Li

Shandong University

Huanjie Li

Shandong University

Wenjuan Li

Shandong University

Jihui Jia

Shandong University

Shaohua Ge

Shandong University

Yundong Sun ( $\square$ syd@sdu.edu.cn )

Shandong University

\section{Research Article}

Keywords: Helicobacter pylori, Oral lichen planus, saliva, microbiota, inflammatory factor

Posted Date: January 4th, 2021

DOl: https://doi.org/10.21203/rs.3.rs-135416/v1

License: (c) (i) This work is licensed under a Creative Commons Attribution 4.0 International License.

Read Full License

Version of Record: A version of this preprint was published at BMC Microbiology on April 20th, 2021. See the published version at https://doi.org/10.1186/s12866-021-02188-0. 


\section{Abstract}

Background Oral lichen planus (OLP), a common clinical oral disease, is associated with an increased risk for malignant transformation. The mechanism underlying the pathogenesis of OLP is unknown. Oral dysbacteriosis is reported to be one of the etiological factors for OLP. Although H. pylori infection is associated with various oral diseases, the correlation between $H$. pylori infection and OLP is unclear. This study aimed to investigate the effect of $H$. pylori infection on OLP pathogenesis and oral microbiome composition in the Chinese population, which has a high incidence of $H$. pylori infection.

Result In this study, the saliva samples of 30 patients with OLP (OLP group) and 21 negative controls (NC group) were collected. H. pylori infection was detected using the carbon-14 urea breath test. The saliva samples were divided into the following four groups based on the $H$. pylori infection status: $H$. pyloripositive OLP (OLP+), H. pylori-positive NC (NC+), H. pylori-negative OLP (OLP-), and H. pylori-negative NC (NC-) groups. The oral microbiome composition was significantly different between the OLP and NC groups and between the OLP- and OLP+ groups. Compared with those in the OLP-group, the incidence of erosive OLP and the salivary levels of cytokines were higher in the OLP+ group. In contrast, the oral microbiome composition and cytokine levels were not significantly different between the NC- and NC+ groups.

Conclusions This is the first report demonstrated that $H$. pylori infection is significantly correlated with the pathogenesis of erosive OLP. The alleviation of $H$. pylori infection may decrease the incidence of erosive OLP.

\section{Background}

The infection from Helicobacter pylori, which is classified as a primary carcinogen by the World Health Organization (WHO), can lead to the development of chronic gastritis, gastric ulcer, and gastric cancer[1, 2]. The human oral cavity is the temporary storage site for $H$. pylori and an extragastric $H$. pylori reservoir[3]. H. pylori infection was detected in the dental plaques and saliva using various molecular techniques $[4,5]$. Additionally, $H$. pylori infection is associated with oral diseases, such as periodontitis and recurrent oral ulcer[5, 6].

The prevalence of oral lichen planus (OLP), a common chronic inflammatory oral mucosal disease, is $0.5-2.2 \%$ in general adult population[7]. The two main subtypes of OLP are reticular OLP and erosive OLP. The reticular type OLP is characterized by protruding white lacy lesions or papules[7, 8], while the erosive type OLP is characterized by erythema, erosion, or ulcerative lesions[7, 8]. OLP can cause different degrees of discomfort, including prolonged burning sensation, bleeding, tingling, and abnormal taste. Previous studies have demonstrated that OLP, which is defined as a precancerous condition by the WHO, is associated with an increased risk for malignant transformation[9].

Various factors, such as immunological, psychological, infectious, and genetic factors are reported to be involved in the pathogenesis of $\operatorname{OLP}[8,10,11]$. Recent studies have demonstrated that oral microbiota is 
involved in the development of oral diseases[12]. The oral microbiota of patients with OLP has increased microbial diversity, decreased Streptococcus abundance, and increased Bacteroides abundance[13-15]. Oral dysbacteriosis is reported to be one of the etiological factors of OLP[16].

The correlation between $H$. pylori infection and OLP has not been elucidated. H. pylori infection has been detected in the periodontal pocket of patients with OLP. Additionally, the presence of $H$. pylori in the oral cavity is associated with leukoplakia and OLP oral lesions[6]. One study reported the possible connection between $H$. pylori and erosive OLP[17]. However, recent studies have reported that $H$. pylori infection was not detected in the mucosal biopsies of patients with OLP, which indicated the lack of correlation between OLP and H. pylori infection[18].

Hence, the studies evaluating the correlation between OLP and H. pylori have reported contradictory findings. Additionally, most of these studies involved the Western population. In the Chinese population, the average $H$. pylori infection rate is high (more than 50\%)[19]. However, the correlation between $H$. pylori and OLP has not been examined in the Chinese population.

In this study, the saliva samples collected from normal control volunteers and patients with OLP were grouped based on the $H$. pylori infection status. The clinical subtypes of OLP in H. pylori-positive and $H$. pylori-negative patients were determined. The findings of this study indicated that $H$. pylori infection increases the incidence of erosive OLP. However, the underlying mechanism was not elucidated. So, the oral microbiome composition of OLP patients with and without $H$. pylori infection was examined to evaluate the effect of $H$. pylori infection on the oral microbiome composition.

\section{Results}

\section{Patients with OLP exhibit enhanced prevalence of $H$. pylori infection}

The saliva samples of 30 patients with OLP (OLP group) and 21 normal control volunteers (NC group) were collected to analyze the correlation between $\mathrm{H}$. pylori infection status and OLP clinical subtypes. Age and gender were not significantly different between the OLP and NC groups (Table 1).

H. pylori infection in the OLP and NC groups was detected using the urease breath test. The prevalence of H. pylori infection in the OLP group (70\%) was markedly higher than that in the NC group (50\%)(Table 1).

Erosive OLP was characterized by a red erosive mucosal surface (Figure S1 in Additional file). The lesion severity and the risk of malignant transformation in erosive OLP are higher than those in reticular OLP[9]. In the OLP group, the incidence of erosive OLP in H. pylori-positive patients (61.9\%) was significantly (Chisquare test) higher than that in $H$. pylori-negative patients $(11.1 \%)(p=0.0041)$. Additionally, H. pylori infection was correlated with the OLP subtypes (Table 2).

\section{Patients with OLP exhibit altered oral microbiome composition}


Previous studies have demonstrated that the oral microbiome composition in patients with OLP was different from that in normal control individuals[20-22]. This study demonstrated that the a diversity of oral microbiota in the OLP group was significantly higher than that in the NC group (Fig.1A and B). Principal coordinate analysis (PCOA) revealed that the $\beta$ diversity of oral microbiota in the OLP group was significantly different from that in the NC group (Fig. 1C).

The oral microbiome composition was significantly different between the OLP and NC groups at the phylum (Fig. 1D) and genus levels (Fig. 1E). The dominant phyla in the oral microbiota were Firmicutes, Proteobacteria, and Bacteroidetes (Fig. 1D). Compared with the NC group, the OLP group exhibited a significantly decreased abundance of Firmicutes and a significantly increased abundance of Bacteroidetes (Fig. 1D). At the genus level, compared with the NC group, the OLP group exhibited a decreased abundance of Streptococcus and increased abundances of Neisseria, Prevotella, and Prevotella7 (Fig. 1E).

The bacterial genera with an average relative abundance higher than $1 \%$ in the oral microbiome of the OLP and NC groups are listed in Fig. 2A. The volcano plot was used to represent the differences in the bacterial composition at the genus level between the OLP and NC groups (Fig. 2B). Bacteria with relative abundance greater than $1 \%$ and significant differences in relative abundance were screened out (Fig. 2B). Compared with the NC group, the OLP group exhibited decreased relative abundances of Streptococcus and Rothia and increased relative abundances of Alloprevotella, Prevotella, Fusobacterium, and Porphyromonas (Fig. 2B and C).

\section{H. pylori infection alters the salivary microbiome composition in patients with OLP.}

To analyze the effect of $H$. pylori infection on the salivary microbiome composition of the OLP and NC groups, the saliva samples were divided into the following four groups based on the H. pylori infection status: OLP+ $(n=21) ;$ OLP- $(n=9) ; N C+(n=10) ; N C-(n=11)$.

The a diversity of salivary microbiota in the OLP+ group was significantly higher than that in the OLPgroup (Fig. 3A, B, C, D and E). In contrast, a diversity was not significantly different between the NC+ and NC- groups (Fig. 3A, B, C, D, and E). PCoA revealed that the $\beta$ diversity of salivary microbiota was significantly different between the OLP+ and OLP- groups. However, the $\beta$ diversity of salivary microbiota was not significantly different between the NC+ and NC- groups (Fig. 3F and G). Additionally, the bacterial composition at the genus and phylum levels was not significantly different between the NC+ and the NC- groups (Figure S2 in Additional file).

The salivary microbiome composition was significantly different between the OLP+ and OLP- groups at the phylum and genus levels (Fig. 4A and B). The predominant bacterial phyla were Proteobacteria, Firmicutes, and Bacteroidetes (Fig. 4A), the relative abundance of Bacteroidetes was significantly high in the OLP+ group. At the genus level, the relative abundance of Alloprevotella in the OLP+ group was significantly higher than that in the OLP- group (Fig. 4B). 
The bacteria in the salivary microbiota of the OLP+ and OLP- groups with an average relative abundance higher than $1 \%$ are listed in Fig. 4C. The volcano plot was constructed to determine the differences in the bacterial composition between the OLP+ and the OLP- groups at the genus level (Fig. 4D). The bacteria with relative abundance higher than $1 \%$ and significant differences in the relative abundance were screened out (Fig. 4D). Compared with the OLP- group, the OLP+ group exhibited significantly increased relative abundances of Alloprevotella and Haemophilus and a significantly decreased relative abundance of Actinomyces (Fig. 4D and E).

\section{Comparative analysis of salivary inflammatory factors}

H. pylori infection can induce the gastric mucosa to secrete inflammatory factors, such as IL-6, IL-8, and IL-17[23]. Previous studies have reported the dysregulated expression of various inflammatory factors, such as IL-6, IL-8, IL-17, and TNF-a in patients with OLP[24]. In this study, the salivary levels of IL-6, IL-8, and IL-17 in the OLP and NC groups were analyzed using ELISA. The salivary levels of IL-6, IL-8, and IL-17 in the OLP group were significantly higher than those in the NC group (Fig. 5A).

Next, the effect of $H$. pylori infection on the salivary levels of inflammatory factors in the OLP and NC groups was evaluated. Additionally, the salivary levels of IL-6, IL-8, and IL-17 were comparatively analyzed between the following groups: OLP+ and OLP- groups; NC+ and NC- groups. Compared with those in the OLP- group, the salivary levels of IL-6, IL-8, and IL-17 were significantly upregulated in the OLP+ group (Fig. 5B). However, the salivary levels of IL-6, IL-8, and IL-17 were not significantly different between the $\mathrm{NC}+$ and NC- groups (Fig. 5C).

Next, the correlation between key bacterial genera and inflammatory factors (IL-6, IL-8, and IL-17) was analyzed by constructing the heat map of Spearman's rank correlation coefficients (Fig. 6A and B). In the OLP and NC groups, the abundances of Alloprevotella, Porphyromonas, Fusobacterium, and Prevotella genera were positively correlated with IL-6 and IL-17, while the abundances of Prevotella and Fusobacterium genera were positively correlated with IL-8. Furthermore, the abundances of Streptococcus and Rothia genera were negatively correlated with IL-7, IL-6, and IL-8 (Fig. 6A). In the OLP+ and OLPgroups, the abundances of Alloprevotella and Haemophilus genera were significantly and positively correlated with IL-17, while those of Actinomyces genus were negatively correlated with IL-7, IL-6, and IL-8 (Fig. 6B).

\section{Correlation of salivary microbiome function with key bacterial genera}

PICRUSt was used to predict the metagenome functional content based on 16S rRNA gene sequencing and Kyoto Encyclopedia of Genes and Genomes (KEGG) pathway analysis (Fig. 7). Compared with those in the NC group, the expression levels of genes involved in various metabolic pathways, such as histidine metabolism, phenylalanine metabolism, novobiocin biosynthesis, lipopolysaccharide (LPS) biosynthesis, LPS biosynthesis proteins, biotin metabolism, ubiquinone, and other terpenoid-quinone biosynthesis were upregulated, while those of genes involved in galactose metabolism, phosphotransferase system, and protein kinase were downregulated in the OLP group (Fig. 7A and B). 
Furthermore, the levels of LPS biosynthesis proteins and LPS biosynthesis in the OLP+ group were upregulated when compared with those in the OLP- group (Fig. 7C and D).

LPS can induce inflammatory reaction[25]. PICRUSt analysis predicted that the microbial metabolic pathways involved in the pathogenesis of OLP are LPS biosynthesis proteins and LPS biosynthesis. The correlation between key bacterial genera and these two metabolic pathways was examined by constructing the heat map of Spearman's rank correlation coefficients (Fig. 8A and B). In the OLP and NC groups, the relative abundances of Alloprevotella, Porphyromonas, Fusobacterium, and Prevotella genera were positively correlated, while those of Rothia were negatively correlated with the levels of LPS biosynthesis proteins and LPS biosynthesis (Fig. 8A). In the OLP+ and OLP- groups, the abundances of Alloprevotella and Haemophilus genera were positively correlated, while those of Actinomyces were negatively correlated with the levels of LPS biosynthesis proteins and LPS biosynthesis (Fig. 8B).

\section{Patients with OLP exhibit enhanced prevalence of $H$. pylori infection}

The saliva samples of 30 patients with OLP (OLP group) and 21 normal control volunteers (NC group) were collected to analyze the correlation between $H$. pylori infection status and OLP clinical subtypes. Age and gender were not significantly different between the OLP and NC groups (Table 1).

H. pylori infection in the OLP and NC groups was detected using the urease breath test. The prevalence of H. pylori infection in the OLP group (70\%) was markedly higher than that in the NC group (50\%)(Table 1).

Erosive OLP was characterized by a red erosive mucosal surface (Figure S1 in Additional file). The lesion severity and the risk of malignant transformation in erosive OLP are higher than those in reticular OLP[9]. In the OLP group, the incidence of erosive OLP in H. pylori-positive patients (61.9\%) was significantly (Chisquare test) higher than that in $H$. pylori-negative patients $(11.1 \%)(p=0.0041)$. Additionally, H. pylori infection was correlated with the OLP subtypes (Table 2 ).

\section{Patients with OLP exhibit altered oral microbiome composition}

Previous studies have demonstrated that the oral microbiome composition in patients with OLP was different from that in normal control individuals[20-22]. This study demonstrated that the a diversity of oral microbiota in the OLP group was significantly higher than that in the NC group (Fig.1A and B). Principal coordinate analysis (PCoA) revealed that the $\beta$ diversity of oral microbiota in the OLP group was significantly different from that in the NC group (Fig. 1C).

The oral microbiome composition was significantly different between the OLP and NC groups at the phylum (Fig. 1D) and genus levels (Fig. 1E). The dominant phyla in the oral microbiota were Firmicutes, Proteobacteria, and Bacteroidetes (Fig. 1D). Compared with the NC group, the OLP group exhibited a significantly decreased abundance of Firmicutes and a significantly increased abundance of Bacteroidetes (Fig. 1D). At the genus level, compared with the NC group, the OLP group exhibited a decreased abundance of Streptococcus and increased abundances of Neisseria, Prevotella, and Prevotella7 (Fig. 1E). 
The bacterial genera with an average relative abundance higher than $1 \%$ in the oral microbiome of the OLP and NC groups are listed in Fig. 2A. The volcano plot was used to represent the differences in the bacterial composition at the genus level between the OLP and NC groups (Fig. 2B). Bacteria with relative abundance greater than $1 \%$ and significant differences in relative abundance were screened out (Fig. 2B). Compared with the NC group, the OLP group exhibited decreased relative abundances of Streptococcus and Rothia and increased relative abundances of Alloprevotella, Prevotella, Fusobacterium, and Porphyromonas (Fig. 2B and C).

\section{H. pylori infection alters the salivary microbiome composition in patients with OLP.}

To analyze the effect of $H$. pylori infection on the salivary microbiome composition of the OLP and NC groups, the saliva samples were divided into the following four groups based on the $\mathrm{H}$. pylori infection status: OLP+ $(n=21) ;$ OLP- $(n=9) ; N C+(n=10) ; N C-(n=11)$.

The a diversity of salivary microbiota in the OLP+ group was significantly higher than that in the OLPgroup (Fig. 3A, B, C, D and E). In contrast, a diversity was not significantly different between the NC+ and NC- groups (Fig. 3A, B, C, D, and E). PCoA revealed that the $\beta$ diversity of salivary microbiota was significantly different between the OLP+ and OLP- groups. However, the $\beta$ diversity of salivary microbiota was not significantly different between the NC+ and NC- groups (Fig. 3F and G). Additionally, the bacterial composition at the genus and phylum levels was not significantly different between the NC+ and the NC- groups (Figure S2 in Additional file).

The salivary microbiome composition was significantly different between the OLP+ and OLP- groups at the phylum and genus levels (Fig. 4A and B). The predominant bacterial phyla were Proteobacteria, Firmicutes, and Bacteroidetes (Fig. 4A), the relative abundance of Bacteroidetes was significantly high in the OLP+ group. At the genus level, the relative abundance of Alloprevotella in the OLP+ group was significantly higher than that in the OLP- group (Fig. 4B).

The bacteria in the salivary microbiota of the OLP+ and OLP- groups with an average relative abundance higher than $1 \%$ are listed in Fig. 4C. The volcano plot was constructed to determine the differences in the bacterial composition between the OLP+ and the OLP- groups at the genus level (Fig. 4D). The bacteria with relative abundance higher than $1 \%$ and significant differences in the relative abundance were screened out (Fig. 4D). Compared with the OLP- group, the OLP+ group exhibited significantly increased relative abundances of Alloprevotella and Haemophilus and a significantly decreased relative abundance of Actinomyces (Fig. 4D and E).

\section{Comparative analysis of salivary inflammatory factors}

H. pylori infection can induce the gastric mucosa to secrete inflammatory factors, such as IL-6, IL-8, and IL-17[23]. Previous studies have reported the dysregulated expression of various inflammatory factors, such as IL-6, IL-8, IL-17, and TNF-a in patients with OLP[24]. In this study, the salivary levels of IL-6, IL-8, 
and IL-17 in the OLP and NC groups were analyzed using ELISA. The salivary levels of IL-6, IL-8, and IL-17 in the OLP group were significantly higher than those in the NC group (Fig. 5A).

Next, the effect of $H$. pylori infection on the salivary levels of inflammatory factors in the OLP and NC groups was evaluated. Additionally, the salivary levels of IL-6, IL-8, and IL-17 were comparatively analyzed between the following groups: OLP+ and OLP- groups; NC+ and NC- groups. Compared with those in the OLP- group, the salivary levels of IL-6, IL-8, and IL-17 were significantly upregulated in the OLP+ group (Fig. 5B). However, the salivary levels of IL-6, IL-8, and IL-17 were not significantly different between the $\mathrm{NC}+$ and NC- groups (Fig. 5C).

Next, the correlation between key bacterial genera and inflammatory factors (IL-6, IL-8, and IL-17) was analyzed by constructing the heat map of Spearman's rank correlation coefficients (Fig. 6A and B). In the OLP and NC groups, the abundances of Alloprevotella, Porphyromonas, Fusobacterium, and Prevotella genera were positively correlated with IL-6 and IL-17, while the abundances of Prevotella and Fusobacterium genera were positively correlated with IL-8. Furthermore, the abundances of Streptococcus and Rothia genera were negatively correlated with IL-7, IL-6, and IL-8 (Fig. 6A). In the OLP+ and OLPgroups, the abundances of Alloprevotella and Haemophilus genera were significantly and positively correlated with IL-17, while those of Actinomyces genus were negatively correlated with IL-7, IL-6, and IL-8 (Fig. 6B).

\section{Correlation of salivary microbiome function with key bacterial genera}

PICRUSt was used to predict the metagenome functional content based on 16S rRNA gene sequencing and Kyoto Encyclopedia of Genes and Genomes (KEGG) pathway analysis (Fig. 7). Compared with those in the NC group, the expression levels of genes involved in various metabolic pathways, such as histidine metabolism, phenylalanine metabolism, novobiocin biosynthesis, lipopolysaccharide (LPS) biosynthesis, LPS biosynthesis proteins, biotin metabolism, ubiquinone, and other terpenoid-quinone biosynthesis were upregulated, while those of genes involved in galactose metabolism, phosphotransferase system, and protein kinase were downregulated in the OLP group (Fig. 7A and B).

Furthermore, the levels of LPS biosynthesis proteins and LPS biosynthesis in the OLP+ group were upregulated when compared with those in the OLP- group (Fig. 7C and D).

LPS can induce inflammatory reaction[25]. PICRUSt analysis predicted that the microbial metabolic pathways involved in the pathogenesis of OLP are LPS biosynthesis proteins and LPS biosynthesis. The correlation between key bacterial genera and these two metabolic pathways was examined by constructing the heat map of Spearman's rank correlation coefficients (Fig. 8A and B). In the OLP and NC groups, the relative abundances of Alloprevotella, Porphyromonas, Fusobacterium, and Prevotella genera were positively correlated, while those of Rothia were negatively correlated with the levels of LPS biosynthesis proteins and LPS biosynthesis (Fig. 8A). In the OLP+ and OLP- groups, the abundances of Alloprevotella and Haemophilus genera were positively correlated, while those of Actinomyces were negatively correlated with the levels of LPS biosynthesis proteins and LPS biosynthesis (Fig. 8B). 


\section{Discussion}

The pathogenesis of OLP, a common clinical oral mucosal disease, is unknown[7, 8]. This study aimed to analyze the effect of $H$. pylori infection on the pathogenesis of OLP. The analysis of clinical data indicated that $H$. pylori infection increased the incidence of erosive OLP. Patients with OLP exhibited distinct salivary microbiome composition and enhanced salivary levels of inflammatory factors. H. pylori infection altered the composition and function of salivary microbiota and increased the salivary levels of inflammatory factors in patients with OLP.

Recent studies have demonstrated that the oral microbiome composition in patients with OLP is different from that in normal control individuals[20-22]. Consistent with the findings of previous studies, this study demonstrated that the salivary microbiome composition in patients with OLP was significantly different from that in normal control individuals. Compared with that in normal controls, the diversity of salivary microbiota was significantly higher in patients with OLP, which was consistent with the results of Kun et a〔21].

The salivary microbiome composition at the genus level was comparatively evaluated between the OLP and NC groups. The relative abundance of several bacteria in the salivary microbiota was significantly different in patients with OLP. Streptococcus spp., which is one of the first microbes to colonize the oral cavity, determines the composition of oral microflora[26]. In the oral cavity, Streptococcus produces molecules that inhibit the growth of pathogens[27]. In this study, patients with OLP exhibited a significantly decreased relative abundance of Streptococcus in the salivary microbiota, which can result in the impairment of the growth-inhibitory effects of Streptococcus against oral pathogens.

Porphyromonas spp. and Fusobacterium spp., which are the causative agents of periodontitis, modulate the T-cell-mediated immune responses[12, 28]. Recent studies have suggested that Porphyromonas spp. and Fusobacterium spp. infections can cause oral cancer[29, 30]. The relative abundances of Porphyromonas spp. and Fusobacterium spp. in the salivary microbiota of patients with OLP were significantly high, which suggested that these microbes are involved in the pathogenesis of OLP. Prevotella spp., a member of the oral microbiota, is abundant in patients with periodontal disease and periodontal abscesses[31]. Wang et al[21] and Du et al[32] reported that the relative abundance of Prevotella increased in the salivary microbiota of patients with OLP, which was consistent with the findings of this study. This indicated that Prevotella is an important pathogenic microorganism involved in the development of OLP. Additionally, the relative abundance of Alloprevotella increased in the salivary microbiota of patients with OLP. Alloprevotella increases the levels of $\mathrm{H}_{2} \mathrm{~S}$ in oral microenvironment[33]. $\mathrm{H}_{2} \mathrm{~S}$ is an inflammatory mediator that can promote the expression of pro-inflammatory factors, including IL-6, in the immune cells of humans and mice[34].

In OLP, various exogenous factors are reported to trigger the T lymphocyte-mediated inflammatory immune responses[8]. Previous studies have indicated that the secretion of inflammatory cytokines may play an important role in the OLP-associated immune dysregulation[24]. IL-6 promotes the proliferation of keratinocytes, which promotes epithelial hyperplasia in OLP[35, 36]. The enhanced production of IL-8 may 
impair the tissue repair mechanism of the keratinocytes[37]. Recent studies have demonstrated that the expression of IL-17 was significantly upregulated in the mucosal tissue and peripheral blood of patients with OLP[38-40], which suggested that IL-17 mediates tissue damage in OLP lesions. IL-17 secreted by the keratinocytes at the lesion site of OLP may play a role in recruiting inflammatory cells to the lesion site[40].

In this study, the relative abundances of gram-negative bacteria, such as Porphyromonas, Fusobacterium, Prevotella, and Alloprevotella, were positively correlated with inflammatory cytokines, such as IL-6, IL-8, and IL-17. This indicated that these four bacterial genera mediate the pathogenesis of OLP by promoting inflammation.

The metabolic pathways of salivary microorganisms associated with LPS synthesis were significantly upregulated in patients with OLP and were positively correlated with the relative abundances of Porphyromonas, Fusobacterium, Prevotella, and Alloprevotella. LPS, which exerts immune-stimulatory and pro-inflammatory effects, can promote local inflammatory responses in tissues and upregulate the expression of inflammatory factors[25]. In the epithelial cells, LPS binds to TLR4 to promote the secretion of pro-inflammatory cytokines and chemokines, such as IL-6 and IL-8 through the JNK signaling pathway[41]. Additionally, LPS can stimulate the secretion of Th17-related cytokines, such as IL-17 in the immune cells[42]. Recent studies have demonstrated that LPS inhibits vitamin D receptors in the oral keratinocytes, which impairs the mucosal homeostasis and leads to epithelial barrier damage. Consequently, this may promote the occurrence and development of OLP[43].

This study, for the first time, demonstrated that $H$. pylori infection is significantly correlated with the pathogenesis of erosive OLP. Recent studies have reported that $H$. pylori infection is closely associated with oral diseases, such as periodontitis, aphthous stomatitis, and oral cancer[44-46]. Th17 cell-related pro-inflammatory cytokines, such as IL- 6 and IL-17 are reported to play an important role in $H$. pylori infection-induced inflammation[47-50]. In the gastric mucosa, $H$. pylori infection can promote the secretion of IL-17[47]. IL-17 induces the secretion of IL-8, recruits neutrophils, and aggravates inflammation through the ERK 1/2 MAP kinase pathway[23]. Furthermore, the virulence factor of $H$. pylori can directly promote the secretion of IL-8 in the epithelial cells[51]. H. pylori infection-induced inflammatory cytokines in the stomach can enter the bloodstream and reach the oral cavity to modulate the immune microenvironment in the oral cavity and aggravate the inflammatory response[52].

The dysregulation of T lymphocyte balance may play an important role in the pathogenesis of OLP[8, 24]. The proportion of Th17 cells in the peripheral blood of patients with erosive OLP is significantly higher than that in patients with reticular OLP[38,53]. The analysis of tissues derived from OLP lesions revealed that the erosive OLP lesions contained significantly high proportions of Th17 cells[39]. These results indicate that Th17-related cytokines, such as IL- 6 and IL-17 are involved in the pathogenesis of erosive OLP[39, 53].

We speculated that $H$. pylori-induced IL-17 may spread to the oral cavity through the bloodstream and increase the proportion of Th17 cells, which may contribute to the development of erosive OLP. 
In this study, the salivary levels of IL-6, IL-8, and IL-17 in the OLP + group were higher than those in the OLP - group. This indicated that $H$. pylori infection alters the oral immune microenvironment in patients with OLP, which can affect the microbiome composition and promote the development of diseases[54]. Additionally, H. pylori infection enhanced the synthesis of LPS in the salivary microbiota of patients with OLP, which may be one of the reasons for the increased incidence rate of erosive OLP.

This study demonstrated that the OLP + group exhibited a decreased relative abundance of Actinomyces in the salivary microbiota. Actinomyces spp., which are the core microorganisms of oral cavity[28], are essential for maintaining the balance of bacterial flora. The decreased abundance of dominant bacteria will lead to the dysregulation of microbiome composition and promote the colonization of other bacteria[55]. In this study, the relative abundances of Alloprevotella and Haemophilus were significantly high in the salivary microbiota of the OLP + group. Haemophilus spp., a gram-negative bacillus, is associated with various opportunistic infections[56-58]. Additionally, Haemophilus spp. can activate the macrophages and promote the secretion of pro-inflammatory cytokines, such as IL-6 and IL-8 [59]. Furthermore, Haemophilus spp. infection can induce Th17 cell differentiation and IL-17 secretion, and accelerate the recruitment of neutrophils[60].

In the NC group, H. pylori infection did not enhance the secretion of salivary inflammatory cytokines, as well as the microbial community structure. Previous studies have also reported that gastric $H$. pylori infection does not affect the oral microbiome composition[52]. There are conflicting reports on the colonization of $H$. pylori in the oral cavity. The number of $H$. pylori is low in the oral cavity[61]. A metaanalysis revealed that the number of $H$. pylori in the saliva was less than that in the dental plaque[62]. Chua et al. did not detect $H$. pylori sequences in the oral swab samples[52]. In this study, it was difficult to detect $H$. pylori in the saliva sample of subjects infected with $H$. pylori through 16S rRNA gene sequencing. This indicated that $H$. pylori infection was not enough to alter the oral immune microenvironment but might promote the development of OLP.

In patients with OLP, H. pylori infection may exacerbate the pathological condition of the oral immune microenvironment and disrupt the homeostasis of the salivary microbiota through the interaction with different members of the microbial community[63]. This may decrease the abundance of beneficial bacteria and increase the abundance of pathogenic bacteria in the oral cavity, which contribute to the development of severe clinical subtype of OLP.

The reason for increased incidence rates of erosive OLP in patients with $H$. pylori infection cannot be fully determined based on the findings of this study. This study elucidated the correlation of $H$. pylori infection with the oral microbiome composition and clinical phenotype of OLP. Hence, we hypothesize that the eradication of $\mathrm{H}$. pylori may relieve the clinical symptoms of OLP and reduce the incidence of erosive OLP.

\section{Conclusions}


This study elucidated the correlation of $H$. pylori infection with the oral microbiome composition and clinical phenotype of OLP. We firstly demonstrated that $H$. pylori infection is significantly correlated with the pathogenesis of erosive OLP Hence, we hypothesize that the eradication of $H$. pylori may relieve the clinical symptoms of OLP and reduce the incidence of erosive OLP. However, further clinical studies are needed to verify this hypothesis.

\section{Methods}

\section{Sample collection}

Patients with OLP(OLP group, $\mathrm{n}=30$ ) who underwent comprehensive clinical and histopathological examinations at the Stomatology Hospital of Shandong University were randomly recruited in this study. According to the clinical diagnosis and clinical classification of OLP by WHO (2005)[64], OLP is divided into the following two subtypes: reticular OLP, characterized by a white papular lesion, white reticular lesion, or white plaque; erosive OLP, characterized by erythema and erosive lesions. According to the follow-up clinical examination, age-matched and gender-matched normal control volunteers were recruited (NC group, $n=21$ ). All study subjects signed the informed consent form. The study subjects did not have a history of periodontitis, dental caries, systemic diseases, smoking, and alcohol consumption. Additionally, the subjects had not undergone antibiotic therapy or received OLP treatment before the sample was collected. This study was approved by the Medical Ethics Committee of School of Stomatology of Shandong University (Protocol Number: 20161001).

The saliva samples were collected following the guidelines of the Manual of Procedures for Human Micro-biome Project (http://hmpdacc.org/resources/tools_protocols.php). Briefly, the unstimulated saliva samples were collected from each patient between 8:00 am and 11:00 am in a sterile DNase/RNase-free conical tube. The study subjected did not consume alcohol or food for at least $2 \mathrm{~h}$ before sampling. The samples were transported to the laboratory and stored at $-80^{\circ} \mathrm{C}$ until further use.

H. pylori infection was detected in the OLP and NC groups using the urea breath test (14C-UBT) kit (Headway, Shenzhen, China). Based on the H. pylori infection status, the samples were divided into the

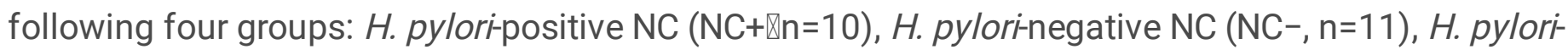
positive OLP $(\mathrm{OLP}+, \mathrm{n}=21)$, and H. pylori-negative OLP $(\mathrm{OLP}-, \mathrm{n}=9)$ groups.

\section{Enzyme linked immunosorbent assay (ELISA)}

The saliva samples $(5 \mathrm{~mL})$ were centrifuged at $4{ }^{\circ} \mathrm{C}$ and $3500 \mathrm{~g}$ for $20 \mathrm{~min}$. The supernatant was stored in a DNase/RNase-free EP tube at $-80^{\circ} \mathrm{C}$ until further use. The concentrations of IL-6, IL-8, and IL-17 were examined using the ELISA kit (Neobioscience Technology Co., Ltd. Shenzhen, China), following the manufacturer's instructions.

\section{DNA extraction and 16S rRNA gene amplification}


The genomic DNA was isolated from the saliva samples using the QIAamp DNA micro kit (Qiagen, Valencia, CA, USA), following the manufacturer's instructions. The V3 and V4 regions (336F-806R) of the $16 S$ rRNA gene were amplified using polymerase chain reaction.

\section{Sequencing and data analysis}

High-throughput sequencing was performed at CloudSeq Biotech, Inc. (Shanghai, China). Briefly, the raw sequence data were obtained from samples sequenced using the Illumina MiSeq sequencer were subjected to base calling and quality filtering. The samples were separated based on the barcode. The adaptors were trimmed and the low-quality reads were removed using Trimmomatic. The paired ends were merged using FLASH. The optimized reads were used for operational taxonomic unit (OTU) clustering and the OTU matrices were generated. The most abundant sequence in each OTU was selected as the representative sequence. The sequences of representative OTU were compared with those listed in the Greengenes database and those of samples. The taxonomic abundance matrices were generated. The determination of alpha and beta diversities, as well as statistical analysis and mapping, were performed using Mother and R environment. The microbial gene function was predicted using PICRUSt.

\section{Statistical analysis}

All data are represented as mean \pm standard deviation. The differences in the cytokine levels between two groups were examined using the $t$-test. The Kruskal-Wallis test was used to analyze the differences in microbiome composition between two groups. The correlation between variables was examined using Spearman's correlation test. All statistical analyses were performed using GraphPad Prism version 7.01 (GraphPad Software, Inc. CA, USA).

\section{Declarations}

\section{Acknowledgements}

We are grateful to Ding Chao doctor from Medical School of Nanjing University for his very kind help in analyzing our data.

\section{Author's Contributions}

YS \& SG: conceptualized and supervised this study; YS \& SL: study design and original draft preparation; WL, YS, JJ \& SG: acquired funding; SL, ZY, JL, HL \& YL: analyzed the data; YS \& SL: involved in writing and editing.

\section{Funding}

This research was subsidized by the National Natural Science Foundation of China (Nos. 81671978, 81471991 and 81772143 ) and the Department of Science and Technology of Shandong Province (Nos. 2018CXGC1208). 


\section{Data availability}

The sequencing data generated in this study are submitted to the Sequence Read Archive of the National Center for Biotechnology Information database (accession number: SRP133987). All data supporting the findings of this study are available in the manuscript. Supplementary material can be obtained from the corresponding author upon reasonable request.

\section{Ethics approval and consent to participate}

The experimental protocol was established, according to the ethical guidelines of the Helsinki Declaration and was approved by the Medical Ethics Committee of School of Stomatology of Shandong University (Protocol Number: 20161001). Written informed consent was obtained from individual or guardian participants.

\section{Consent for publication}

Not applicable.

\section{Conflict of Interest}

The authors declare no conflict of interest.

\section{Author details}

${ }^{1}$ Key Laboratory for Experimental Teratology of Ministry of Education and Department of Microbiology, School of Basic Medical Science, Cheeloo College of Medicine, Shandong University, Jinan, Shandong, 250012, China.

2 Department of Periodontology, School and Hospital of Stomatology, Cheeloo College of Medicine, Shandong University \& Shandong Key Laboratory of Oral Tissue Regeneration \& Shandong Engineering Laboratory for Dental Materials and Oral Tissue Regeneration, Jinan, Shandong 250012, China.

${ }^{3}$ Department of Periodontology, Nanjing Stomatological Hospital, Medical School of Nanjing University, 30 Zhongyang Road, Nanjing, 210008, China.

${ }^{4}$ School of Medicine, Cheeloo College of Medicine, Shandong University, Jinan, Shandong, 250012, China.

\section{References}

1. Camilo V, Sugiyama T, Touati E. Pathogenesis of Helicobacter pylori infection. Helicobacter. 2017;22:1-6.

2. Burucoa C, Axon A. Epidemiology of Helicobacter pylori infection. Helicobacter. 2017;22:1-5. 
3. Al Sayed A, Anand PS, Kamath KP, Patil S, Preethanath RS, Anil S. Oral Cavity as an Extragastric Reservoir of Helicobacter pylori. ISRN Gastroenterol. 2014;2014:1-16.

4. Ansari SA, Iqbal M un N, Khan TA, Kazmi SU. Association of oral Helicobacter pylori with gastric complications. Life Sci. 2018;205 March:125-30. doi:10.1016/j.Ifs.2018.05.026.

5. Matamala-Valdés L, Sánchez-Alonzo K, Parra C, Sáez K, Aguayo-Reyes A, García A. Detection of intracellular helicobacter pylori in candida. SPP from neonate oral swabs. Rev Assoc Med Bras. 2018;64:928-35.

6. Kazanowska-Dygdała M, Duš I, Radwan-Oczko M. The presence of Helicobacter pylori in oral cavities of patients with leukoplakia and oral lichen planus. J Appl Oral Sci. 2016;24:18-23.

7. Alrashdan MS, Cirillo N, McCullough M. Oral lichen planus: a literature review and update. Arch Dermatol Res. 2016;308:539-51.

8. Kurago ZB. Etiology and pathogenesis of oral lichen planus: An overview. Oral Surg Oral Med Oral Pathol Oral Radiol. 2016;122:72-80. doi:10.1016/j.0000.2016.03.011.

9. Tampa M, Caruntu C, Mitran M, Mitran C, Sarbu I, Rusu LC, et al. Markers of oral lichen planus malignant transformation. Dis Markers. 2018;2018:7-10.

10. Sugerman PB, Savage NW, Walsh LJ, Zhao ZZ, Zhou XJ, Khan A, et al. The pathogenesis of oral lichen planus. Crit Rev Oral Biol Med. 2002;13:350-65.

11. Nosratzehi T. Oral lichen planus: An overview of potential risk factors, biomarkers and treatments. Asian Pacific J Cancer Prev. 2018;19:1161-7.

12. Zhang Y, Wang X, Li H, Ni C, Du Z, Yan F. Human oral microbiota and its modulation for oral health. Biomed Pharmacother. 2018;99 January:883-93. doi:10.1016/j.biopha.2018.01.146.

13. Jia G, Zhi A, Lai PFH, Wang G, Xia Y, Xiong Z, et al. The oral microbiota - A mechanistic role for systemic diseases. Br Dent J. 2018;224:447-55.

14. Carvalho MFMS de, Cavalieri D, Do Nascimento S, Lourenço TGB, Ramos DVR, Pasqualin D da C, et al. Cytokines Levels and Salivary Microbiome Play A Potential Role in Oral Lichen Planus Diagnosis. Sci Rep. 2019;9:1-10.

15. Choi YS, Kim Y, Yoon HJ, Baek KJ, Alam J, Park HK, et al. The presence of bacteria within tissue provides insights into the pathogenesis of oral lichen planus. Sci Rep. 2016;6 June:1-13. doi:10.1038/srep29186.

16. Kragelund C, Keller MK. The oral microbiome in oral lichen planus during a 1-year randomized clinical trial. Oral Dis. 2019;25:327-38.

17. Attia EAS, Abdel Fattah NSA, Abdella HM. Upper gastrointestinal findings and detection of Helicobacter pylori in patients with oral lichen planus: Clinical dermatology • Original article. Clin Exp Dermatol. 2010;35:355-60.

18. Hulimavu SR, Mohanty L, Tondikulam N V., Shenoy S, Jamadar S, Bhadranna A. No evidence for Helicobacter pylori in oral lichen planus. J Oral Pathol Med. 2014;43:576-8. 
19. Liu WZ, Xie Y, Lu H, Cheng H, Zeng ZR, Zhou LY, et al. Fifth Chinese National Consensus Report on the management of Helicobacter pylori infection. Helicobacter. 2018;23:1-17.

20. Yu FY, Wang QQ, Li M, Cheng YH, Cheng YSL, Zhou Y, et al. Dysbiosis of saliva microbiome in patients with oral lichen planus. BMC Microbiol. 2020;20:1-12.

21. Wang K, Lu W, Tu Q, Ge Y, He J, Zhou Y, et al. Preliminary analysis of salivary microbiome and their potential roles in oral lichen planus. Sci Rep. 2016;6:1-10. doi:10.1038/srep22943.

22. Li Y, Wang K, Zhang B, Tu Q, Yao Y, Cui B, et al. Salivary mycobiome dysbiosis and its potential impact on bacteriome shifts and host immunity in oral lichen planus. Int J Oral Sci. 2019;11:13. doi:10.1038/s41368-019-0045-2.

23. Bagheri N, Azadegan-Dehkordi F, Shirzad H, Rafieian-Kopaei M, Rahimian G, Razavi A. The biological functions of IL-17 in different clinical expressions of Helicobacter pylori-infection. Microb Pathog. 2015;81:33-8.

24. Pekiner FN, Demirel GY, Borahan MO, Özbayrak S. Cytokine profiles in serum of patients with oral lichen planus. Cytokine. 2012;60:701-6.

25. Maldonado RF, Sá-Correia I, Valvano MA. Lipopolysaccharide modification in gram-negative bacteria during chronic infection. FEMS Microbiol Rev. 2016;40:480-93.

26. Abranches J, Zeng L, Kajfasz JK, Palmer S, Chakraborty B, Wen Z, et al. Biology of Oral Streptococci. Gram-Positive Pathog. 2019;6:426-34.

27. Sitkiewicz I. How to become a killer, or is it all accidental? Virulence strategies in oral streptococci. Mol Oral Microbiol. 2018;33:1-12.

28. Verma D, Garg PK, Dubey AK. Insights into the human oral microbiome. Arch Microbiol. 2018;200:525-40.

29. Zhang W long, Wang S sha, Wang H fan, Tang YJ, Tang $Y$ ling, Liang $X$ hua. Who is who in oral cancer? Exp Cell Res. 2019;384:111634.

30. Malinowski B, W囚sierska A, Zalewska K, Sokołowska MM, Bursiewicz W, Socha M, et al. The role of Tannerella forsythia and Porphyromonas gingivalis in pathogenesis of esophageal cancer. Infect Agent Cancer. 2019;14:1-8.

31. Chen J, Wu X, Zhu D, Xu M, Yu Y, Yu L, et al. Microbiota in Human Periodontal Abscess Revealed by 16S rDNA Sequencing. Front Microbiol. 2019;10 July:1-12.

32. Du G huan, Wang Y feng, Chen J jun, Deng Y wen, Han X zhe, Tang G yao. Potential association between Fusobacterium nucleatum enrichment on oral mucosal surface and oral lichen planus. Oral Dis. 2020;26:122-30.

33. Ye W, Zhang Y, He M, Zhu C, Feng XP. Relationship of tongue coating microbiome on volatile sulfur compounds in healthy and halitosis adults. J Breath Res. 2020;14.

34. Bhatia M. H2S and Inflammation: An Overview. Hand Exp Pharmacol. 2015;230:395.

35. Krueger J, Ray A, Tamm I, Sehgal PB. Expression and function of interleukin-6 in epithelial cells. J Cell Biochem. 1991;45:327-34. 
36. Gu GM, Martin MD, Darveau RP, Truelove E, Epstein J. Oral and serum IL-6 levels in oral lichen planus patients. Oral Surgery, Oral Med Oral Pathol Oral Radiol Endodontology. 2004;98:673-8.

37. Mozaffari HR, Sharifi R, Mirbahari S, Montazerian S, Sadeghi M, Rostami S. A systematic review and meta-analysis study of salivary and serum interleukin-8 levels in oral lichen planus. Postep Dermatologii i Alergol. 2018;35:599-604.

38. Wang H, Han Q, Luo Z, Xu C, Liu J, Dan H, et al. Oral lichen planus may enhance the expression of Th17-associated cytokines in local lesions of chronic periodontitis. Clin Oral Investig. 2014;18:164754.

39. Piccinni MP, Lombardelli L, Logiodice F, Tesi D, Kullolli O, Biagiotti R, et al. Potential pathogenetic role of Th17, Th0, and Th2 cells in erosive and reticular oral lichen planus. Oral Dis. 2014;20:212-8.

40. Monteiro BVDB, Pereira JDS, Nonaka CFW, Godoy GP, Da Silveira ÉJD, Miguel MCDC. Immunoexpression of Th17-related Cytokines in Oral Lichen Planus. Appl Immunohistochem Mol Morphol. 2015;23:409-15.

41. Zhang LL, Chen B, Fan XY, Wu SS, Zhang SQ, Wu HM. LPS Cooperates with Poly-L-Arginine to Promote IL-6 and IL-8 Release via the JNK Signaling Pathway in NCl-H292 Cells. J Immunol Res. 2016;2016.

42. Okuyama H, Tominaga A, Fukuoka S, Taguchi T, Kusumoto Y, Ono S. Spirulina lipopolysaccharides inhibit tumor growth in a Toll-like receptor 4-dependent manner by altering the cytokine milieu from interleukin-17/interleukin-23 to interferon-y. Oncol Rep. 2017;37:684-94.

43. Zhao B, Li R, Yang F, Yu F, Xu N, Zhang F, et al. LPS-induced Vitamin D Receptor Decrease in Oral Keratinocytes Is Associated with Oral Lichen Planus. Sci Rep. 2018;8:1-9.

44. Zarić S, Bojić B, Janković L, Dapčeví B, Popović B, Čakić S, et al. Periodontal therapy improves gastric helicobacter pylori eradication. J Dent Res. 2009;88:946-50.

45. Dayama A, Srivastava V, Shukla M, Singh R, Pandey M. Helicobacter pylori and Oral Cancer: Possible association in a preliminary case control study. Asian Pacific J Cancer Prev. 2011;12:1333-6.

46. Adler I, Muiño A, Aguas S, Harada L, Diaz M, Lence A, et al. Helicobacter pylori and oral pathology: Relationship with the gastric infection. World J Gastroenterol. 2014;20:9922-35.

47. Lehours P, Ferrero RL. Review: Helicobacter: Inflammation, immunology, and vaccines. Helicobacter. 2019;24:1-6.

48. Butz AM. Helicobacter: Inflammation, immunology, and vaccines Alice. Physiol Behav. 2017;176:139-48.

49. Arachchi PS, Fernando N, Weerasekera MM, Senevirathna B, Weerasekera DD, Gunasekara CP. Proinflammatory Cytokine IL-17 Shows a Significant Association with Helicobacter pylori Infection and Disease Severity. Gastroenterol Res Pract. 2017;2017.

50. Ikuse T, Blanchard TG, Czinn SJ. Inflammation, immunity, and vaccine development for the gastric pathogen helicobacter pylori. Curr Top Microbiol Immunol. 2019;421:1-19. 
51. Jang S, Kim J, Cha JH. Cot kinase plays a critical role in Helicobacter pylori-induced IL-8 expression. J Microbiol. 2017;55:311-7.

52. Chua EG, Chong JY, Lamichhane B, Webberley KM, Marshall BJ, Wise MJ, et al. Gastric Helicobacter pylori infection perturbs human oral microbiota. PeerJ. 2019;2019:1-14.

53. Vered M, Fürth E, Shalev Y, Dayan D. Inflammatory cells of immunosuppressive phenotypes in oral lichen planus have a proinflammatory pattern of expression and are associated with clinical parameters. Clin Oral Investig. 2013;17:1365-73.

54. Graves DT, Corrêa JD, Silva TA. The Oral Microbiota Is Modified by Systemic Diseases. J Dent Res. 2019;98:148-56.

55. Netuschil NBA and L, Abstract 4. The Oral Microbiota. 2016;902:5-32. doi:10.1007/978-3-31931248-4.

56. Murphy TF, Brauer AL, Sethi S, Kilian M, Cai X, Lesse AJ. Haemophilus haemolyticus: A Human Respiratory Tract Commensal to Be Distinguished from Haemophilus influenzae. J Infect Dis. 2007;195:81-9.

57. Mukundan D, Ecevit Z, Patel M, Marrs CF, Gilsdorf JR. Pharyngeal colonization dynamics of Haemophilus influenzae and Haemophilus haemolyticus in healthy adult carriers. J Clin Microbiol. 2007;45:3207-17.

58. Kistler JO, Arirachakaran P, Poovorawan Y, Dahlén G, Wade WG. The oral microbiome in human immunodeficiency virus (HIV)-positive individuals. J Med Microbiol. 2015;64:1094-101.

59. Berenson CS, Murphy TF, Wrona CT, Sethi S. Outer membrane protein p6 of nontypeable Haemophilus influenzae is a potent and selective inducer of human macrophage proinflammatory cytokines. Infect Immun. 2005;73:2728-35.

60. Essilfie AT, Simpson JL, Horvat JC, Preston JA, Dunkley ML, Foster PS, et al. Haemophilus influenzae infection drives IL-17-mediated neutrophilic Allergic airways disease. PLoS Pathog. 2011;7.

61. Yee JKC. Are the view of helicobacter pylori colonized in the oral cavity an illusion? Exp Mol Med. 2017;49.

62. Anand PS, Kamath KP, Anil S. Role of dental plaque, saliva and periodontal disease in Helicobacter pylori infection. World J Gastroenterol. 2014;20:5639-53.

63. Baker JL, Bor B, Agnello M, Shi W, He X. Ecology of the Oral Microbiome: Beyond Bacteria. Trends Microbiol. 2017;25:362-74.

64. Van der Meij EH, Van der Waal I. Lack of clinicopathologic correlation in the diagnosis of oral lichen planus based on the presently available diagnostic criteria and suggestions for modifications. J Oral Pathol Med. 2003;32:507-12.

\section{Tables}


Table 1 Demographic and clinical parameters of the saliva study samples found no statistically significant differences in age and gender of each group.

\begin{tabular}{llllll} 
Characteristics & NC- & NC+ & OLP- & OLP+ & p value \\
\cline { 2 - 5 } & $(\mathbf{n = 1 0 )}$ & $(\mathbf{n = 1 1 )}$ & $(\mathbf{n = 9})$ & $(\mathbf{n = 2 1 )}$ & \\
Age (mean $\pm S D)$ & $46.44 \pm 3.772$ & $48.30 \pm 3.211$ & $48.30 \pm 3.211$ & $46.71 \pm 2.618$ & $0.45^{[1]}$ \\
Male/female & $7 / 3$ & $11 / 0$ & $8 / 1$ & $16 / 5$ & $0.5^{[2]}$ \\
Smoking habit; & 0 & 0 & 0 & 0 & \\
Alcohol drinking; & 0 & 0 & 0 & 0 & \\
Antibiotics usage; & 0 & 0 & 0 & 0 &
\end{tabular}

${ }^{[1]}$ ANOVA; ${ }^{[2]}$ Fisher's test.

Table 2 Chi-sauared test showed statistically significant association of infection of $H$. Pylori and the alteration of OLP subtype $(p<0.005)$.

\begin{tabular}{|c|c|c|c|}
\hline \multirow[t]{2}{*}{ OLP subtype (\%) } & OLP- & OLP+ & \multirow[t]{2}{*}{$p$ value } \\
\hline & $(n=9)$ & $(n=21)$ & \\
\hline Reticular OLP $(n=16)$ & $8(88.9)$ & $8(38.1)$ & \multirow[t]{2}{*}{$0.0041^{[1]}$} \\
\hline Erosive OLP $(n=14)$ & $1(11.1)$ & 13 (61.9) & \\
\hline
\end{tabular}

\section{Figures}



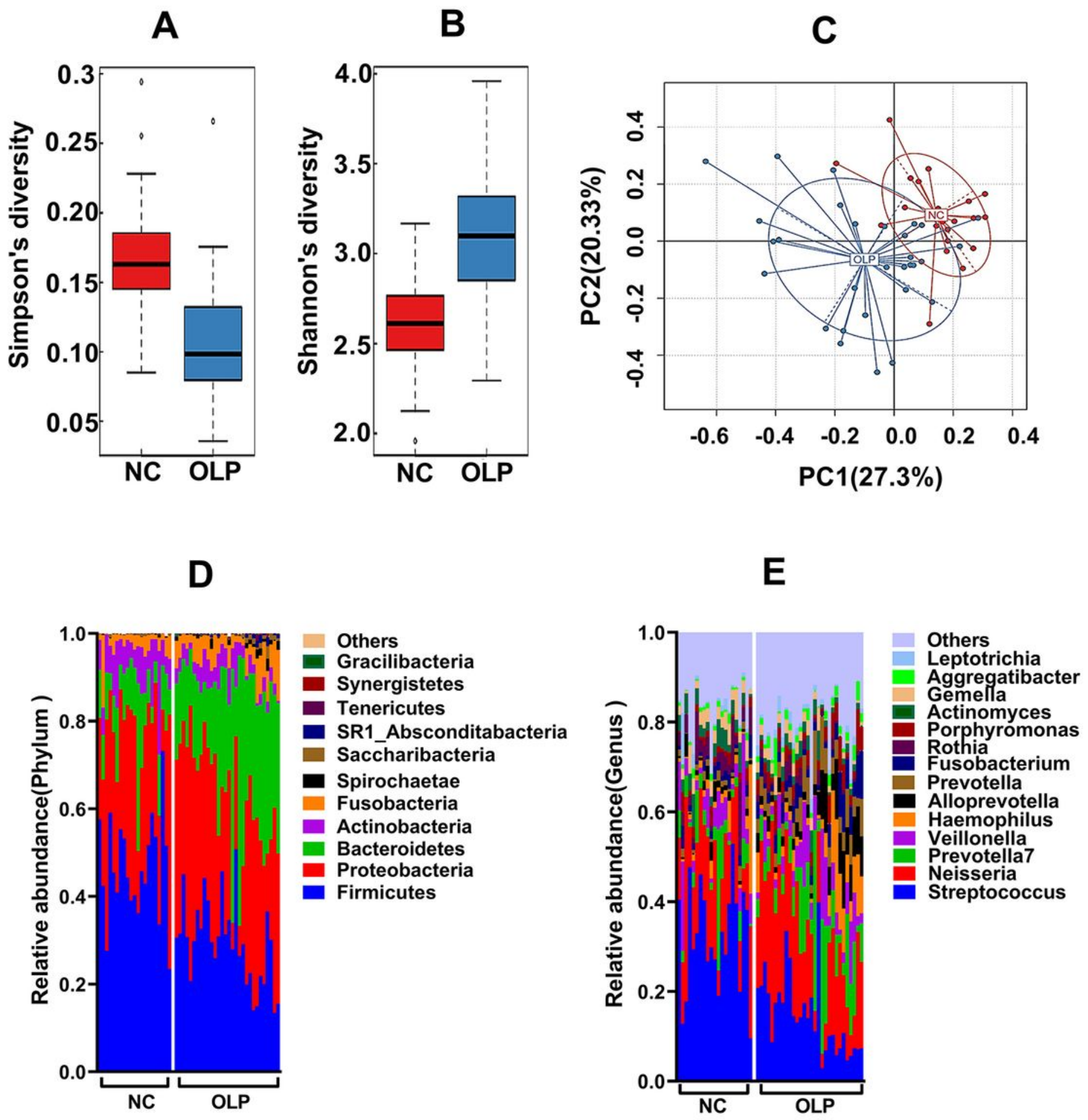

Figure 1

Comparison of the salivary microbiome profiles between the NC $(n=21)$ and OLP groups $(n=30)$. (A) Simpson's diversity index of salivary microbiome in the NC and OLP groups $(p=0.00003)$. (B) Shannon's diversity index of salivary microbiome in the NC and OLP groups $(p=0.0002)$. (C) Principal coordinate analysis (PCoA) plots of unweighted UniFrac distances of oral microbiota in the NC and OLP groups. 
Comparative analysis of the oral microbiome composition at the phylum (D) and genus (E) levels between the NC and OLP groups.
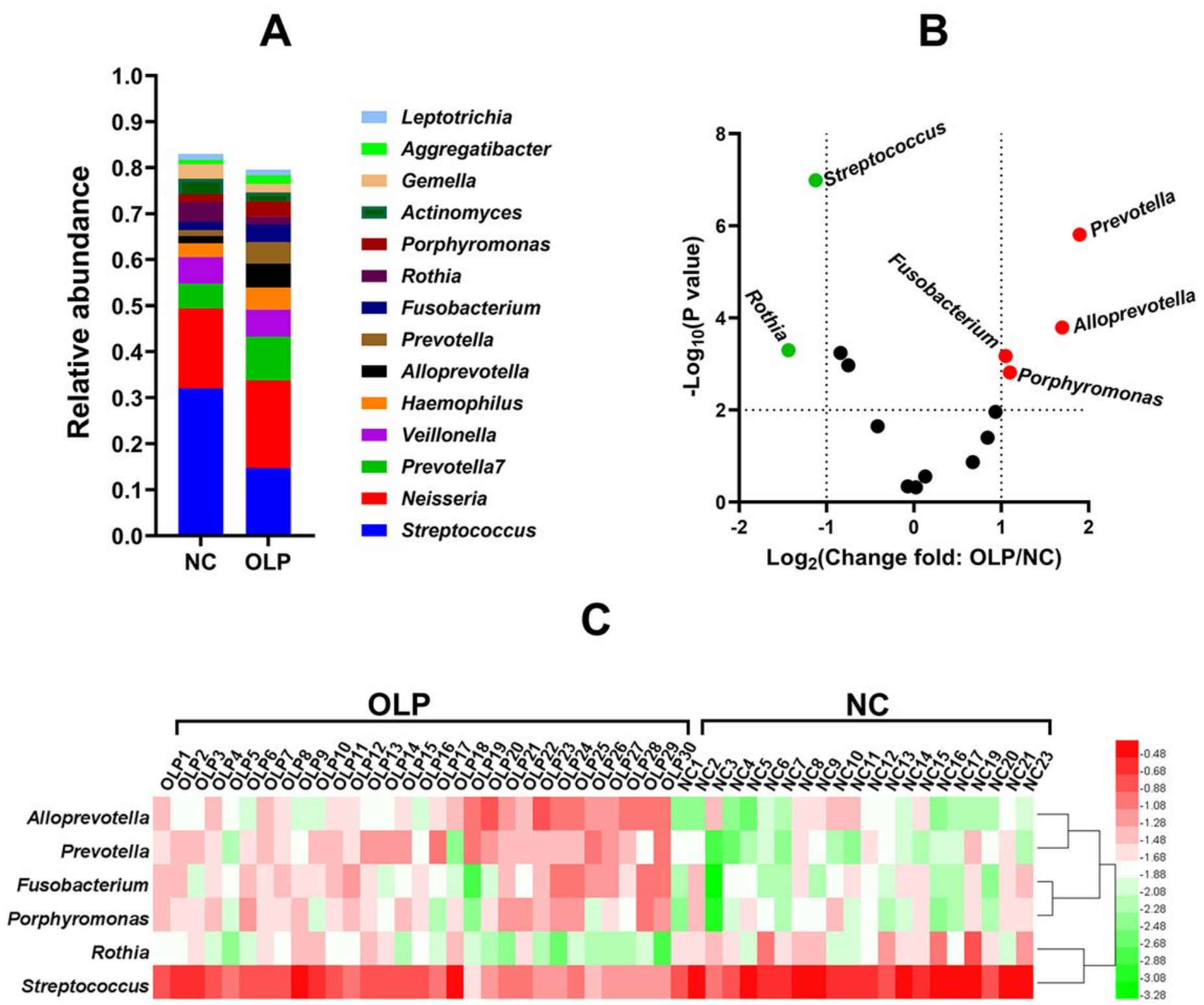

Figure 2

Comparison of the salivary microbiome composition at the genus level between the NC and OLP groups. (A) Comparative analysis of the salivary microbiome composition between the NC ( $n=21)$ and OLP $(n=30)$ groups (average relative abundance $>1 \%$ in the two groups). (B) Volcano plot analysis of salivary microbiome composition between the NC $(n=21)$ and OLP $(n=30)$ groups. The $p$-value was calculated using the t-test. According to the results shown in panel $(A)$, bacteria with a relative abundance greater than $1 \%$ were selected and marked as key bacteria. (C) Heat map showing the relative abundances of the key bacteria for sampled individuals of the community subgroups. 

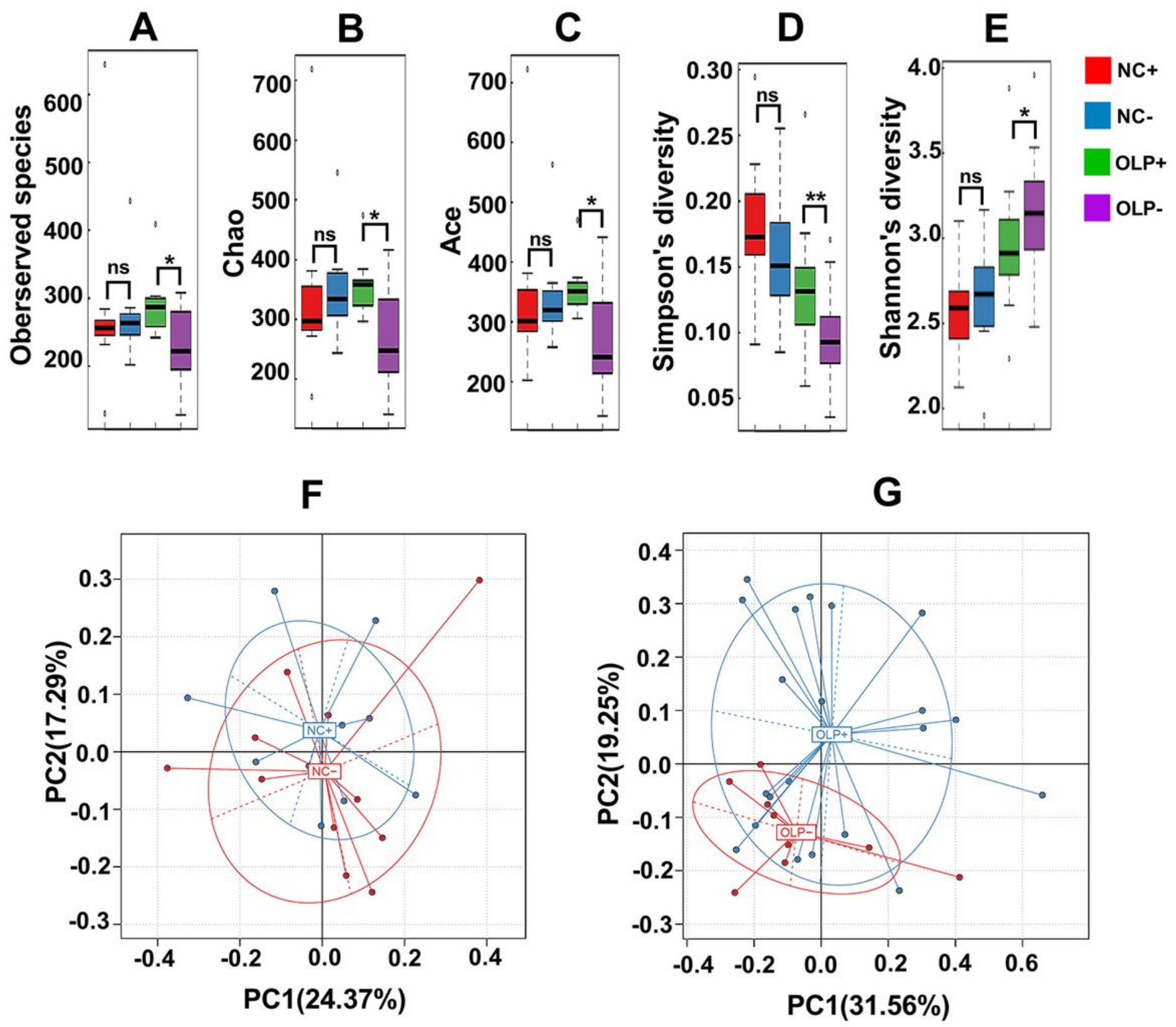

Figure 3

Comparison of the salivary microbiome composition between the NC-, NC+, OLP-, and OLP + groups. (A) Observed species, (B) Chao diversity index, (C) Ace diversity index, (D) Simpson's diversity index, and (E) Shannon's diversity index between the NC- $(n=11)$ and NC+ $(n=10)$ groups, as well as between the OLP$(n=9)$ and OLP+ $(n=21)$ groups. Principal coordinate analysis (PCoA) plots of unweighted UniFrac distances of saliva microbiota between the NC- $(n=11)$ and NC+ $(n=10)$ groups $(F)$ and between the OLP- $(n=9)$ and OLP+ $(n=21)$ groups $(G) .{ }^{*} p<0.05 ;{ }^{*} p<0.01 ; n s$, no significance. 
A

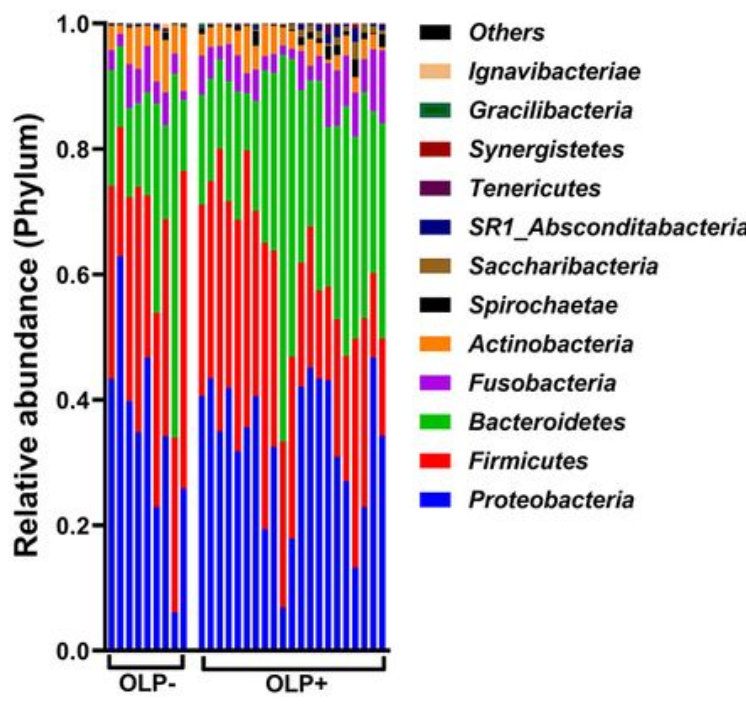

C

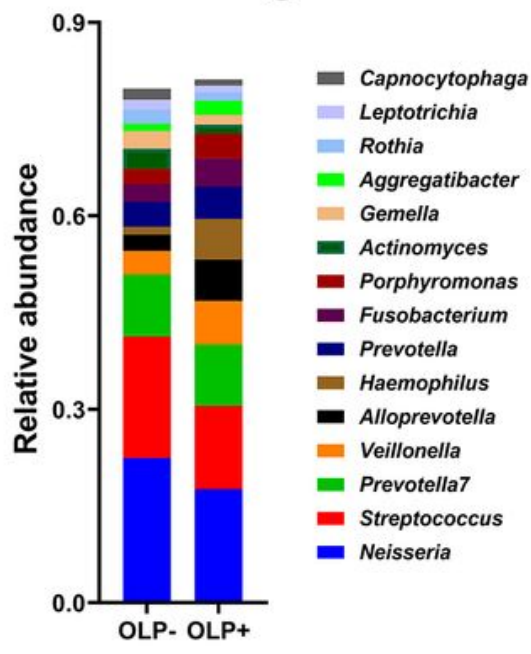

B

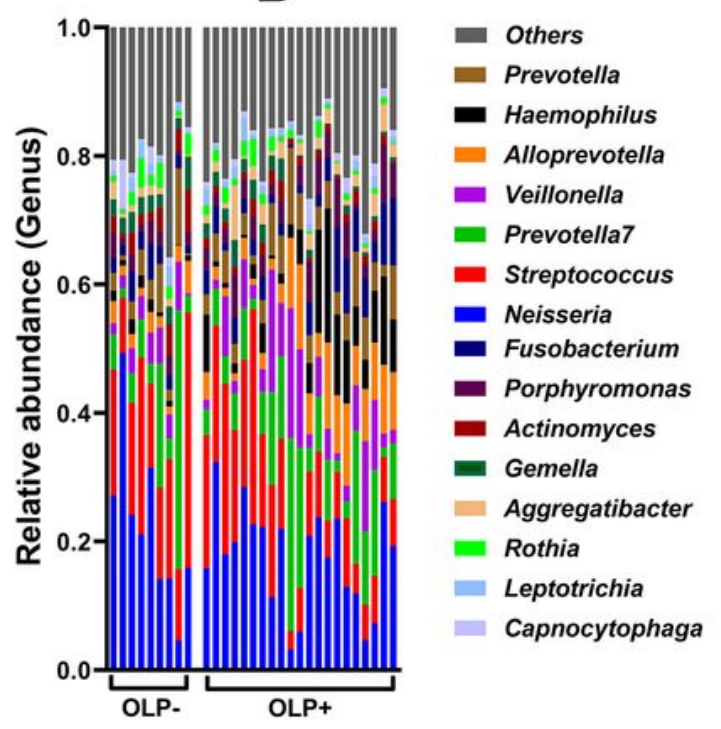

D

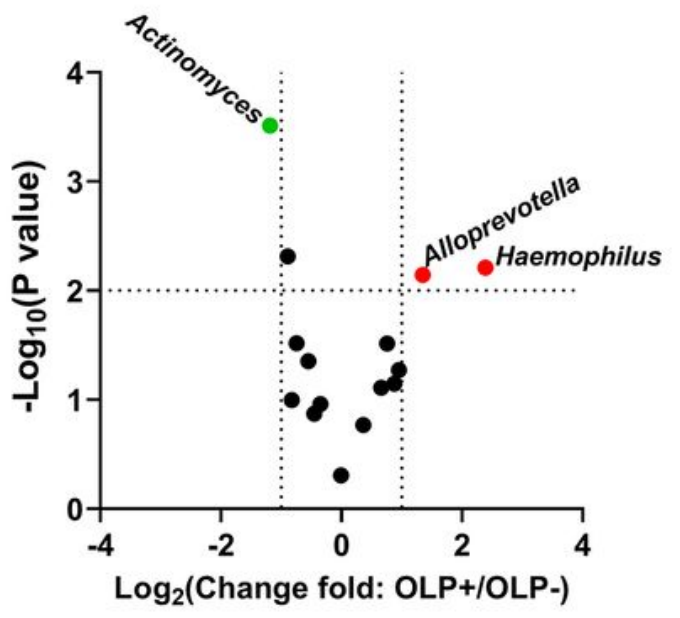

$\mathbf{E}$

OLP-

OLP+

Alloperetellla
Heamophilus
Actinomyces

Figure 4

Comparative analysis of the salivary microbiome composition between the OLP- $(n=9)$ and OLP+ $(n=21)$ groups. (A) Comparative analysis of the salivary microbiome composition at the phylum and (B) the genus levels between the OLP- and OLP + groups. (C) Comparative analysis of the salivary microbiome composition between the OLP- and OLP+ groups (average relative abundance $>1 \%$ in the two groups). (D) Volcano plot analysis of salivary microbiome composition between the OLP- and OLP+ groups. The 
p-value was calculated using the t-test. According to the results shown in panel (C), bacteria with a relative abundance greater than $1 \%$ were selected and marked as key bacteria. (E) Heat map showing the relative abundance of the key bacteria for sampled individuals of the community subgroups.
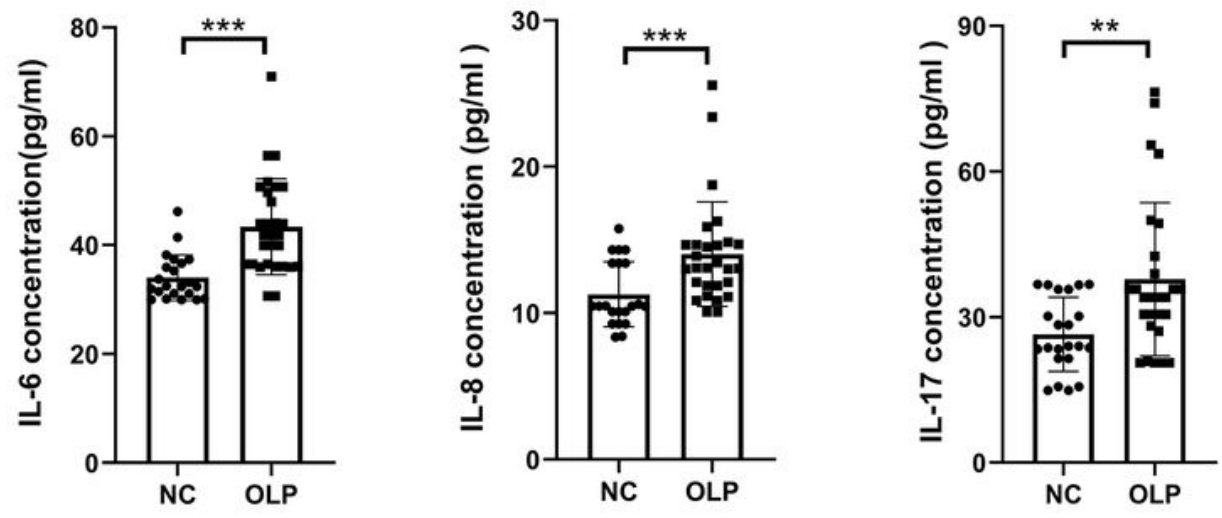

B
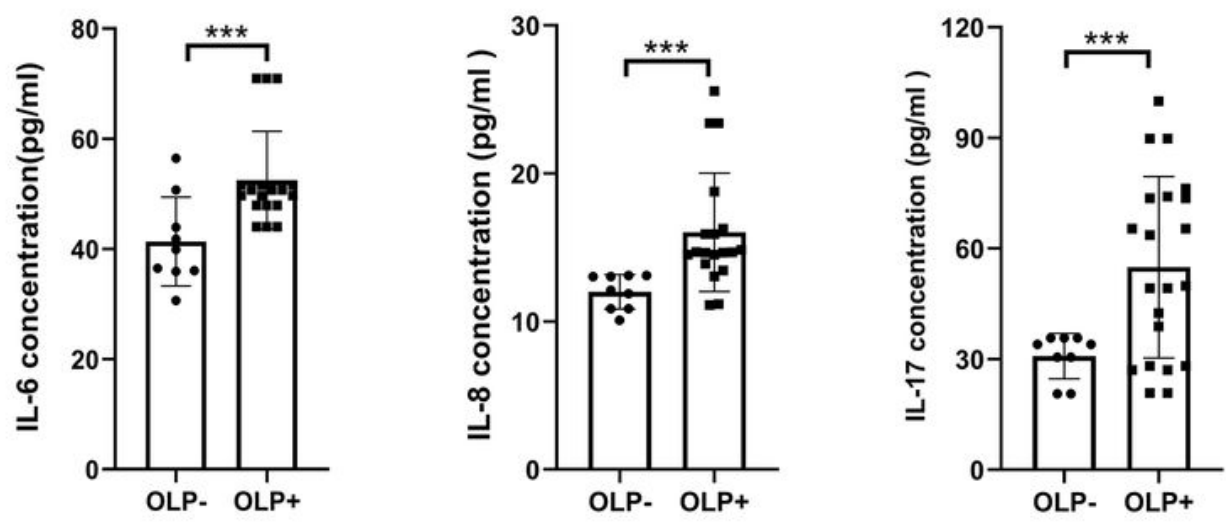

\section{C}
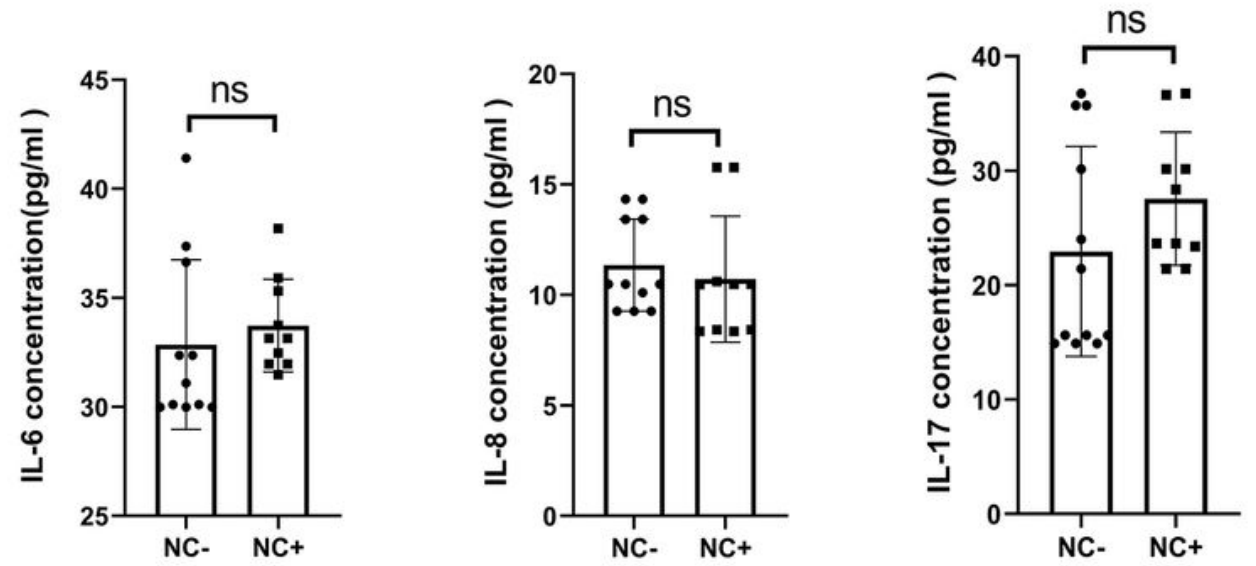

Figure 5

The salivary levels of cytokines (IL-6, IL-8, and IL-17) were measured using ELISA. Comparative analysis of the salivary cytokine levels between the following groups: NC $(n=21)$ and OLP $(n=30)$ groups (A); OLP- 
$(n=9)$ and OLP+ $(n=21)$ groups $(B) ; N C-(n=11)$ and NC+ $(n=10)$ groups (C). ${ }^{*}<<0.05 ;{ }^{* *} p<0.01 ; * \star * p<$ 0.001 ; ns, no significance.

A

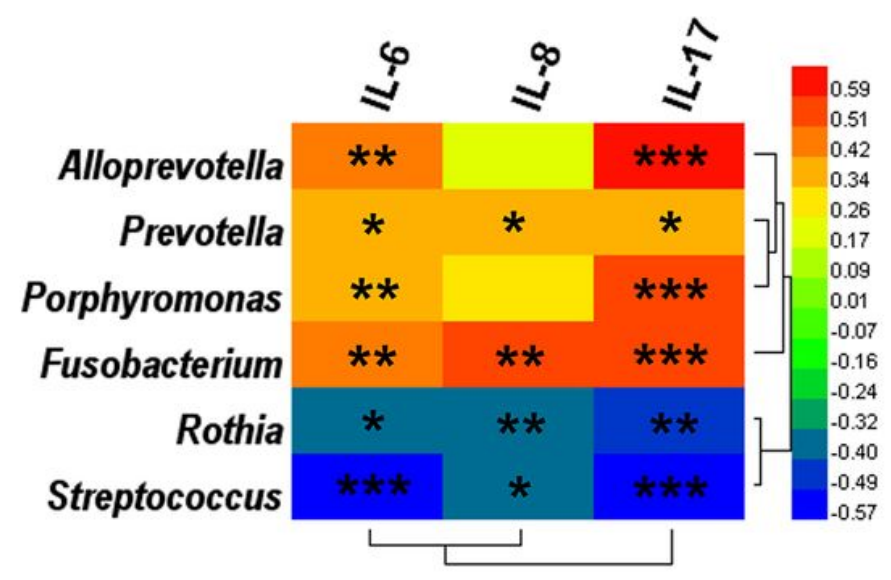

B

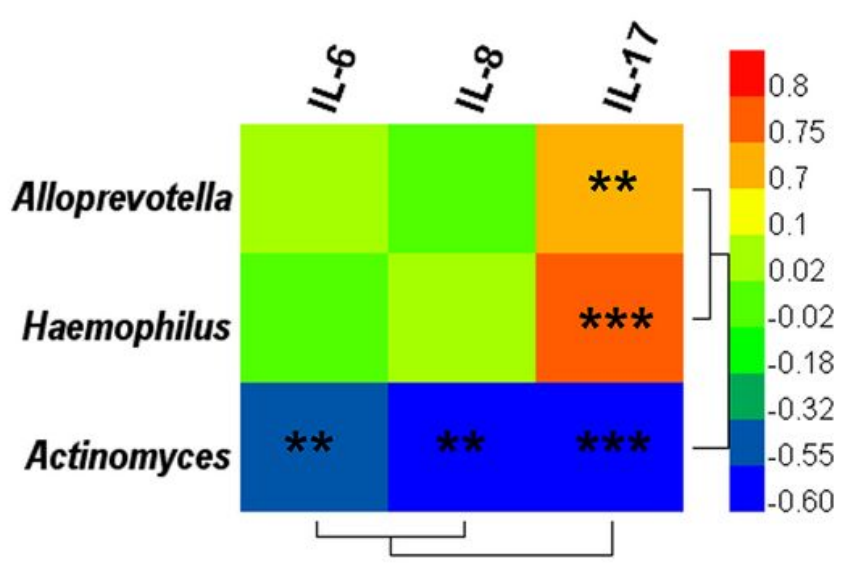

Figure 6

Correlation of cytokine production with the relative abundance of salivary key bacteria at the genus level . (A) NC $(n=21)$ and OLP $(n=30)$ groups; $(B)$ OLP- $(n=9)$ and OLP+ $(n=21)$ groups. The color of cells represents the correlation coefficient $(R)$ values. Cells marked with an asterisk show significance after

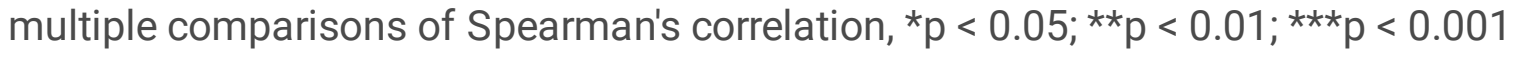




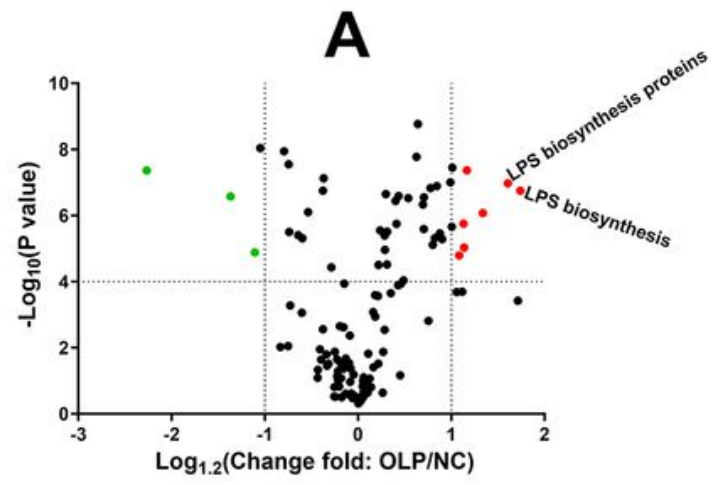

\section{B}

$\square$ NC $\square$ OLP
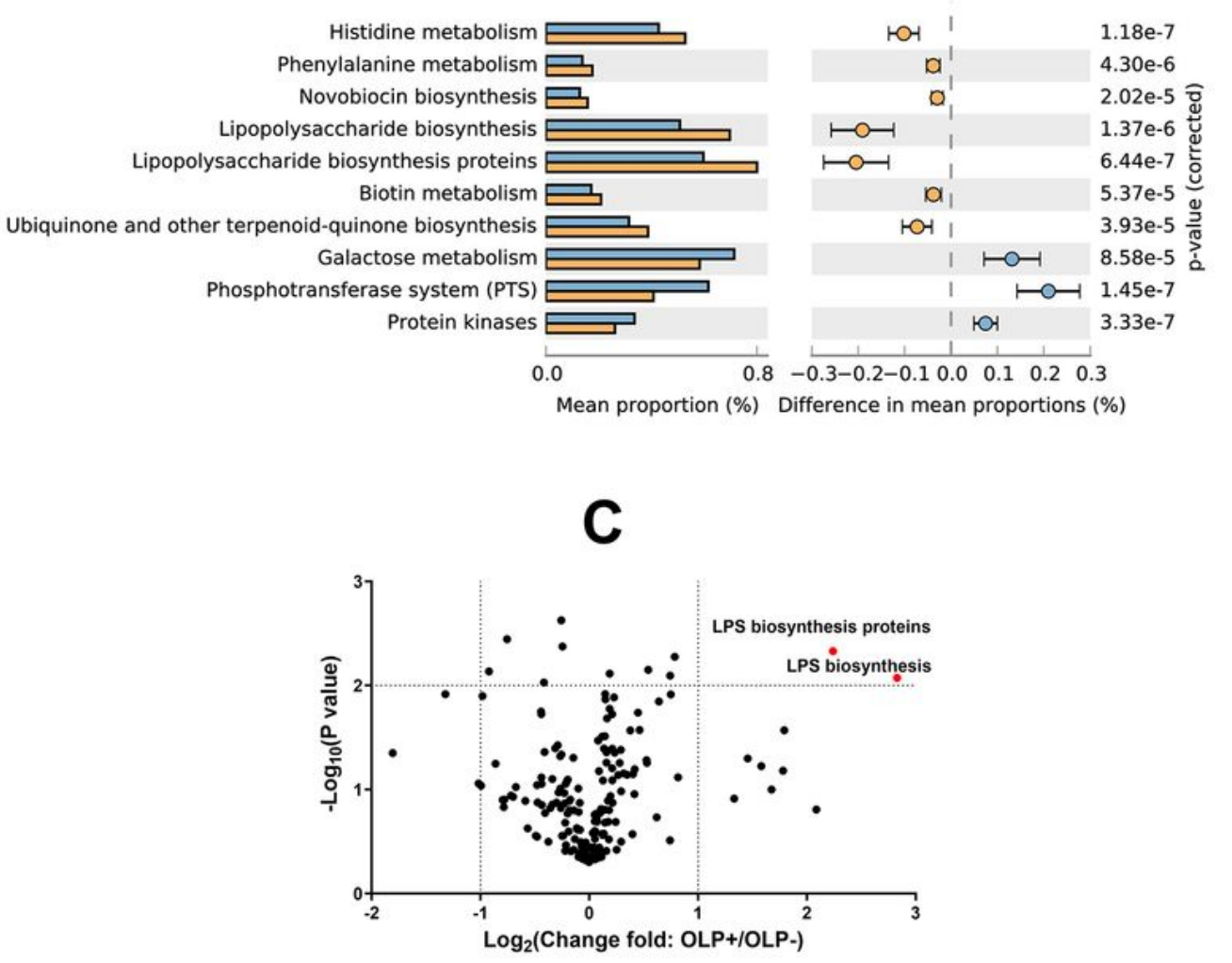

D

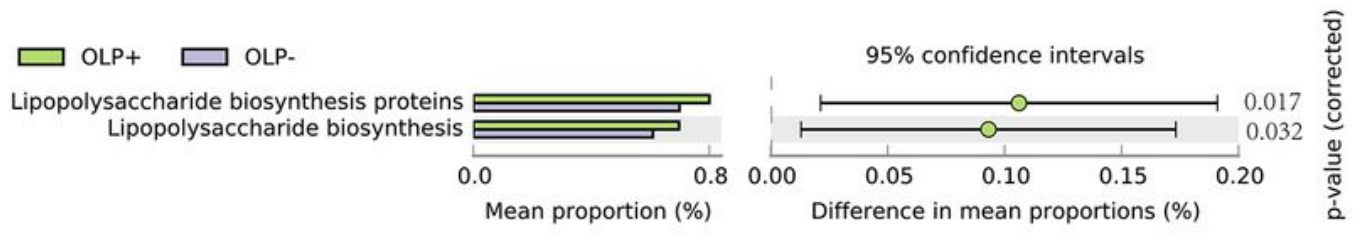

\section{Figure 7}

Comparative analysis of the relative abundance of PICRUSt-generated functional profiles of salivary microbiota. Volcano plot analysis of altered Kyoto Encyclopedia of Genes and Genomes (KEGG) pathways between the following groups: NC $(n=21)$ and OLP $(n=30)$ groups $(A)$; OLP- $(n=9)$ and OLP+ $(n=21)$ groups $(C)$. KEGG pathways with an average relative abundance greater than $2 \%$ in the two groups were included. The p-value was calculated using the two-tailed paired t-test (B) and (D) based on volcano 
plot analysis (Figure $\mathrm{A}$ and Figure $\mathrm{C}$ ). The distinct gene categories were selected according to significant differences in gene categories at level 3 (t-test, $p<0.05$ ). The bar plots on the left show the mean proportion of each KEGG pathway. The dot plots on the right show the differences in mean proportions between the two indicated groups using p-values.
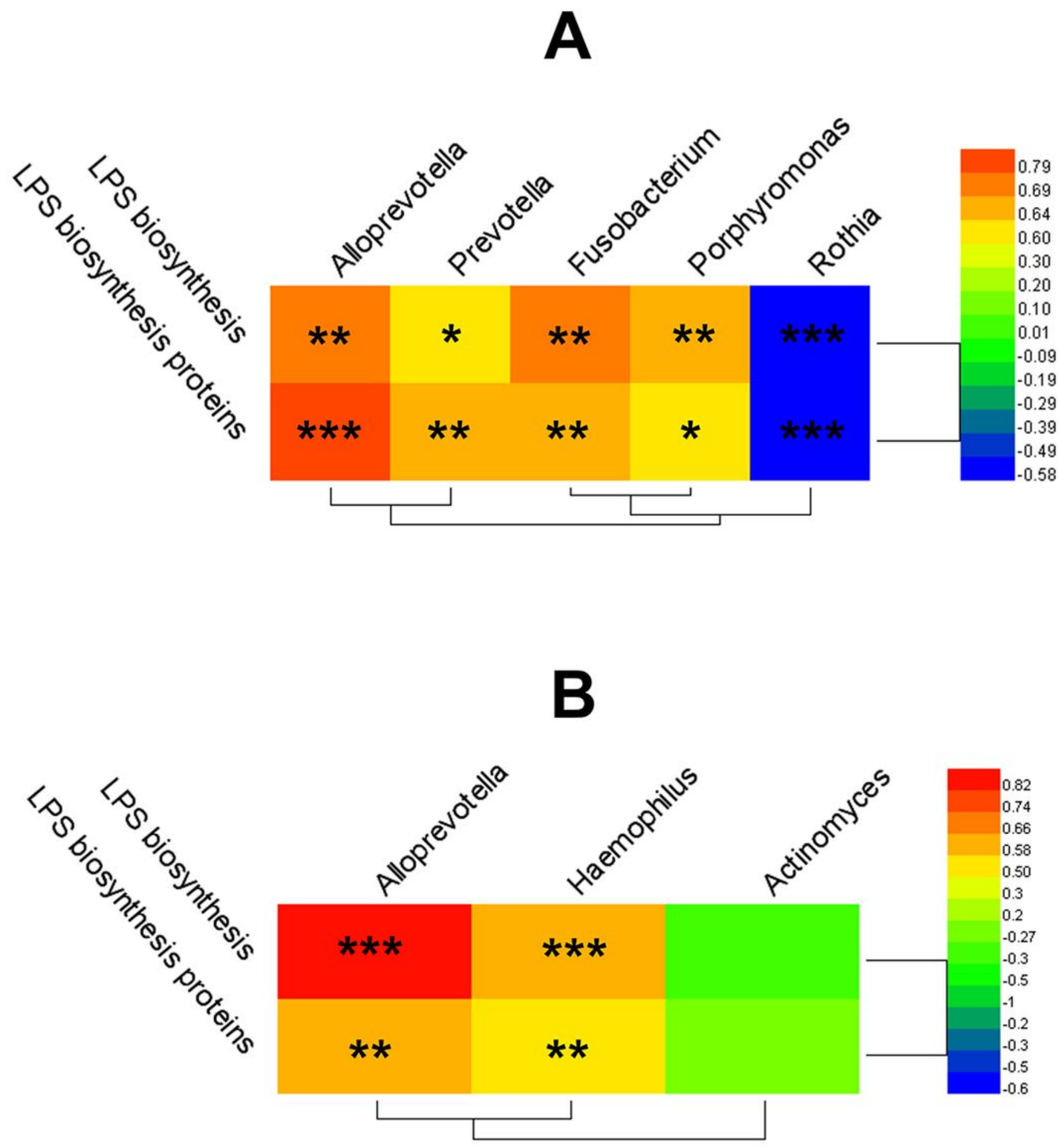

Figure 8 
Correlation of PICRUSt-generated key pathway with the relative abundance of salivary key bacteria at the genus level. (A) NC $(n=21)$ and OLP $(n=30)$ groups; (B) OLP- $(n=9)$ and OLP+ $(n=21)$ groups. The color of cells represents the correlation coefficient $(R)$ values. Cells marked with an asterisk show significance after multiple comparisons using Spearman's correlation, ${ }^{*} p<0.05 ;{ }^{* \star} p<0.01 ; * \star p<0.001$.

\section{Supplementary Files}

This is a list of supplementary files associated with this preprint. Click to download.

- additionfile.docx 